\title{
GLCM and PCA Algorithm based Watermarking Scheme
}

\author{
Jyoti Juneja \\ M.Tech Computer Science Student \\ DAV Institute of Engineering \& Technology, \\ Jalandhar, India
}

\author{
Avani Chopra \\ Assistant Professor \\ DAV Institute of Engineering \& Technology, \\ Jalandhar, India
}

\begin{abstract}
Digital watermarking is the mechanism by which security is provided to the sensitive data which is stored in the databases in the form images. In this process, all the essential features of an image is extracted and calculated after which this original image is implanted into the watermark image. In this research paper, GLCM and PCA algorithm has been utilized in order to improve the working capability of the neural network based watermarking technique. Therefore, the features of the original images are extracted with the help of GLCM and PCA algorithm. The scaling factor defines the output of the PCA algorithm which is used for implementation. On the basis of simulation results it is concluded that proposed algorithm performs well in terms of PSRN and MSE and MATLAB tool is used for the implementation of the proposed method.
\end{abstract}

\section{Keywords \\ GLCM, PCA, PSRN, MSE, Scaling Factor}

\section{INTRODUCTION}

Digital watermarking is the technique in which all the information is embedded into digital multimedia so that it cannot be utilized or copied by anyone. Various purposes are fulfilled by this process such as broadcast monitoring, data authentication, data indexing and many more [1]. This technique must satisfy such as broadcast monitoring, data authentication, data indexing and many more. This digital watermarking system must satisfy trade-offs between conflicting needs of sensory activity transparency, knowledge capability and strength against attacks. Hence for the investigation of these trades-offs an information-theoretic perspective has been utilized [2]. There are two categories of roles in the process of watermark such as, in first, watermark is considered as a transmission code and to recover whole transmitted data correctly decoder must be used. Second, the watermark serves as a verification code.

\subsection{Purpose of Digital Watermarking}

The invisible watermarks would be recognized by the resource, writer, originator, owner, and dispenser or authorized consumer of a document or image. The main objective of this technique is to protect the content of the image permanently, without changing so that no unauthorized user can copy or edit the content. If there is any illicit usage, this watermark provides facilitation to the owner to claim its copyright. This technique also facilitate various functionalities such as it trace images when it is utilized by unauthorized user [3]. This technique must satisfy such as broadcast monitoring, data authentication, data indexing and many more. Therefore, with the help of digital watermarking it becomes possible to mark each image uniquely for every buyer.

\subsubsection{Visible Watermarks}

The extension of the concept of logos is defined as the visible watermarks. These watermarks are applicable to images only.
These logos are embedded within an image but they are transparent in nature. By cropping the center part of the image, these watermarks cannot be removed from an image. These watermarks are protected against attacks such as statistical analysis [3].

\subsubsection{Invisible watermark}

The content in the invisible watermark is hidden and cannot be detected by any unauthorized agency. These watermarks have been utilized for content and author authentication and unauthorized copying [4].

\subsubsection{Public watermark}

The specialized algorithm is used to read or retrieved such watermarks. Therefore, public watermarks are not secure to use and useful for carrying IPR information. To the labels they are good alternative [5].

\subsubsection{Fragile watermark}

Fragile watermarks are also known as tamper-proof watermarks. By data manipulation such watermarks are destroyed.

\subsubsection{Private Watermark}

In order to read or retrieve private watermarks, it is necessary to have a secret key. These watermarks are also known as the secure watermarks.

\subsubsection{Perceptual watermarks}

A perceptual watermark utilizes the features of human sensory system in order to provide the invisible but robust watermark. They are also known as the transparent watermarks as they provide extremely high quality contents.

\subsubsection{Bit-stream watermark}

It is the watermarking which is utilized for the compression of data such as video.

\subsection{Algorithms for Watermarking:}

\section{Singular Value Decomposition (SVD):}

In the linear algebra, Singular Value Decomposition (SVD) plays an essential role and an important topic for many mathematicians. It contains various practical and theoretical values and its special features are it can be utilized for any real $(\mathrm{m}, \mathrm{n})$ matrix. Let's assume a matrix A with $\mathrm{m}$ rows and $\mathrm{n}$ columns, with rank $\mathrm{r}$ and $\mathrm{r} \leq \mathrm{n} \leq \mathrm{m}$, after which factorization of A can be done into three matrices such as:

$$
\mathrm{A}=\mathrm{USV}^{\mathrm{T}}
$$

In the least square sense has the optimal matrix decomposition in the SVD as it integrates the whole signal energy into few coefficients. In order to split the system into a set of linearly independent components, an effective and efficient method is utilized which is known as Singular value decomposition. Each system, in the SVD has its own energy contribution. In the numerical analysis this method is used as numerical technique which is utilized to diagonalizable each matrix. Due to its vast advantages, it is an attractive algebraic transform 
method used for image processing such as maximum energy packing, which is used to manipulate the bases of image into two distinctive subspaces i.e. data and noise subspaces. These subspaces utilized in the noise filtering and in various application of watermarking. The key properties of the SVD are developed by each of these applications and also solve the least squares problem.

SVD is one of the types of orthogonal matrix decomposition method which is feasible and robust. In the area of signal processing, this method is become more popular due to the conceptual and stability reasons of SVD. In the image processing, this method is unique in the transformation of algebraic and it has also an essential properties in imaging. Only some of the properties of SVD are utilized in the in image processing as other needs more evaluation. For the image, several properties of SVD are highly beneficial such as maximum energy packing, solving of least squares problem, pseudo- inverse of a matrix computation and multivariate analysis. The relation to the rank of a matrix is the key property of SVD as its ability to approximate matrices. Digital images can also be represented by low rank matrices as it can be described by sum of small set of Eigen images.

\section{DWT in Image Processing}

Discrete wavelet transform (DWT) is the part of the linear transformation in which all the operations are performed on a data vector which transforms it into the same length of different vector numerically. Data is separated into different frequency components by using this tool and each component is studied in order to match resolution to its scale. Cascade filtering is used for the computation of DWT which has factor 2 for sub sampling. In various application of signal processing, the Discrete Wavelet Transform (DWT) has been utilized. An image or video is decomposed by 2-D discrete wavelet transform (DWT) into sub-images, 3 details and 1 approximation. The originality of an image matches with the approximation of sub-image which is $1 / 4$ the scale of the original. In both the horizontal and the vertical directions 2-D DWT is an application of the 1-D DWT. In an image, the frequency band is separated by DWT into a various detail components such as sub-band (LL) as well as horizontal (HL), vertical $(\mathrm{LH})$ and diagonal $(\mathrm{HH})$. With the help of wavelet decomposition, the watermarks are embedded in low frequency in order to increase the robustness of attacks.

The watermark embedded into high frequency sub-bands makes the watermark more invisible as the HVS is less sensitive to high frequencies. When it is embedded into low frequencies, it makes the more robust system against a variety of attacks. For the processing of non-stationary signals this method is useful. Wavelets are defined as the transformation of the small waves in which frequency varies for limited time period. In an image both frequency and spatial description is provided by the Wavelet transform. In this transformation the temporal information is retained, unlike conventional Fourier transform. Using mother wavelet, it is possible to create wavelet by conversions and dilations of a fixed function. The signal can be splits into high and low frequency parts using DWT. The information about the edge components is stored in the high frequency while in case of low frequency part signals are again splits into high and low frequency parts. For the watermarking, high frequency components are utilized as the sensitivity of human eye is less to edge changes. For the decomposition of each level in the two dimensional application, DWT is performed in the vertical direction after which in the horizontal direction. There are 4 sub-bands: LL1, LH1, HL1, and HH1 after the first level of decomposition. The LL sub band of the previous level is utilized as the input for the decomposition of each successive level. DWT is applied to LL1 band for the second level decomposition in which LL1 band is decomposed into the four sub-parts. For the decomposition of third level, DWT is applied to LL2 band which decompose this band into the four sub-bands - LL3, LH3, HL3, HH3. This process results in 10 sub-bands per component. The highest frequency bands LH1, HL1, and HH1 are present in the image and the lowest frequency band is contained in the LL3. In the signal processing, DWT is utilized in wide variety of applications such as compression of audio and video, noise removal from audio and wireless antenna distribution for simulation. The energy of Wavelets are concentrated in time and for the analysis of transient, time-varying signals, they are well suited method as real life signals are time varying in nature.

\section{LITERATURE SURVEY}

Proposed in this paper a robust image watermarking technique which is used for the protection of the copyright on the basis of 3-level discrete wavelet transforms (DWT). Various parameters has been utilized such as peak-signal-to-noiseratio (PSNR) and mean square error (MSE), for the comparison of proposed method with the 1-level and 2-level DWT based image watermarking technique [6].

Presented a novel multipurpose watermarking scheme in this paper, is used for the copyright protection and content authentication. In this watermarking scheme, fragile and robust watermarks are embedded simultaneously [9]. The wavelet coefficients of a host image are quantized as masking threshold units (MTUs) for which cocktail watermarking was utilized to add two complementary watermarks which can be extracted without access to the host image.

Presented for the computer analysis and machine vision, Image Processing Algorithms are the basics fundamentals explained in this paper. Various image processing algorithms are analyzed in this paper by their logical classification. The application of such techniques is illustrated by the specific methods that are interlinked with real world images [10]. More than one method is utilized in most of the cases. For different messages this is basis for the comparison as positive and negative characteristics of each technique is described.

Presented in handling digital multimedia there are two major problems such as authenticity \& copyright protection which is required to minimize [11]. Various watermarking techniques were compared in this paper using DWT and DWT-DCT methods. The performance of the proposed method was analyzed in terms of PSNR Similarity factor of watermark and recovered watermark.

Presented due to the rapid growth of networked multimedia systems, there is proliferation of digitized media nowadays [4]. This advancement in the technology has created need to develop new methods for the copyright protection of multimedia objects by enforcing new technologies. Therefore, one such technology that protects the digital images from illegal exploitations is the Digital image watermarking that has been developed. These are based on the discrete wavelet transform method and considered as the widely used method in various applications as compared to others.

Presented a robust watermarking technique in this paper utilized for color and grayscale image. Various techniques have been involved in the proposed method that confirms the security and robustness of watermarking. In the 3rd level of DWT method, this proposed technique of watermarks was implemented. For the security reasons, this watermark image 
is passed through chaotic encryption process before its implementation. In order to minimize the influence of original image, this proposed method used the DCT (Discrete Cosine Transform) with special coefficient shifting algorithm [7].

\section{PROPOSED METHODOLOGY}

Watermarking is considered as the effective and efficient technique which provides security to the image data stored in the databases. There are two types of watermarking techniques such as blind and semi-blind watermarking techniques. The machine learning technique, the OS-ELM

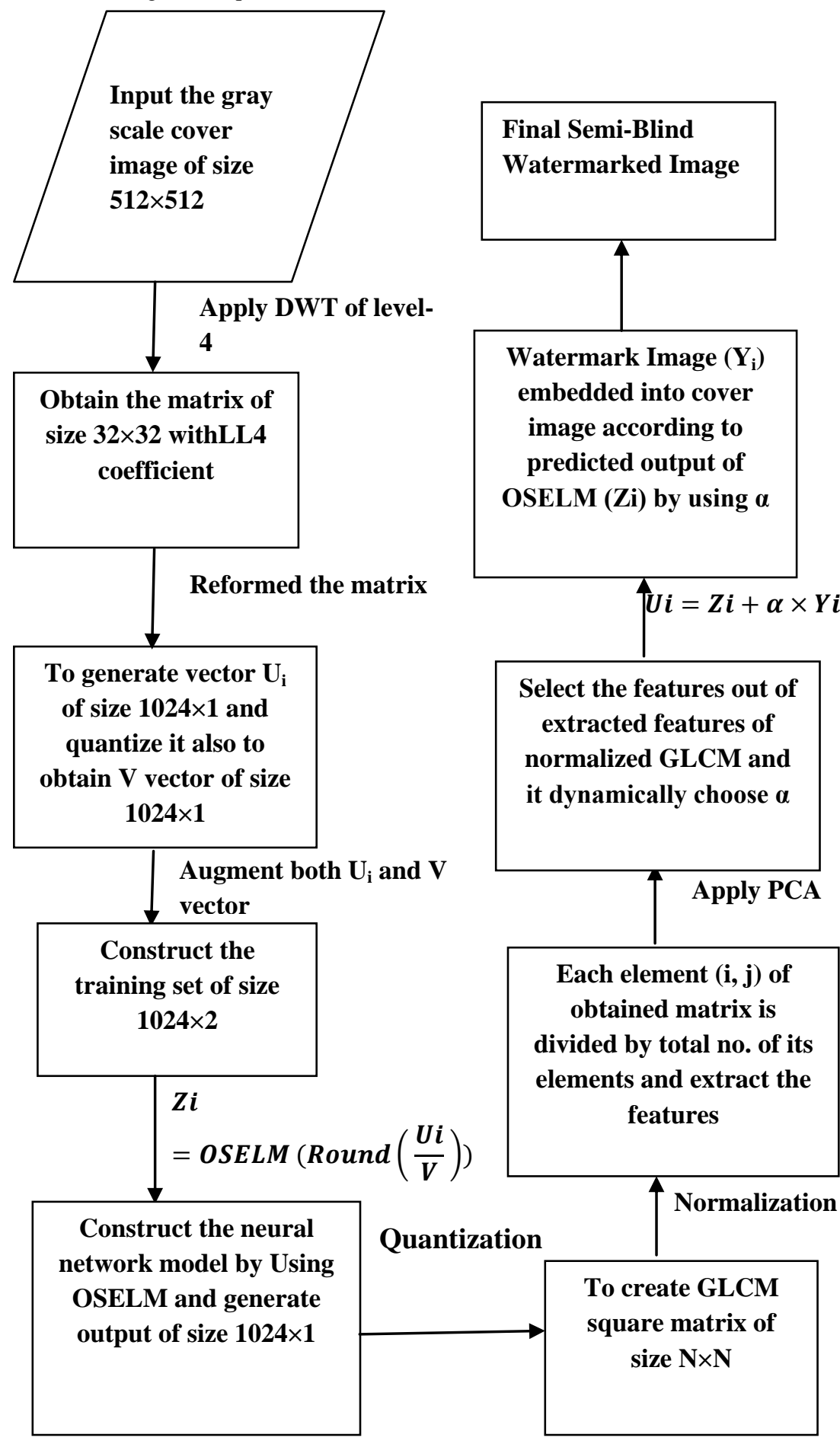

technique was utilized in this paper for the generation of the semi-blind watermarked image. The features of the original image and watermark image are extracted by applying four levels DWT technique. For the generation of the semi-blind watermarks, the training images was utilized as input which is analyzed with the DWT algorithm in order to produce final training sets. The textual features of the images are analyzed with the help of DWT algorithm, with the help of GLCM algorithm. This algorithm is utilized as it is less complex in nature and optimal for the generation of training set for the

arked Image 
The proposed algorithm can be applied in the following steps:-

1. Input the gray scale cover image of size $512 \times 512$.

2. Apply DWT of level-4 to obtain matrix of size $32 \times 32$ with LL4 coefficients.

3. Reformed the obtained image into vector $\mathrm{U}_{\mathrm{i}}$ of size $1024 \times 1$.

4. Now augment the $U_{i}$ vector with its quantized vector $V$ of same size in order to construct the training set of size $1024 \times 2$.

5. Then construct the neural network model by using OSELM to generate an output of size $1024 \times 1$ by using equation 2 .

$$
Z i=\operatorname{OSELM}\left(\operatorname{Round}\left(\frac{U i}{V}\right)\right) .
$$

6. Quantize the data of image to obtain the pixels in order to create GLCM square matrix of size $\mathrm{N} \times \mathrm{N}$.

7. Normalize the GLCM i.e. each element $(i, j)$ of matrix is divided by its total number of elements then obtained elements are considered as probabilities of finding relations and extract the texture features.

8. Now use the PCA algorithm to select the features from the extracted features of step 7and it dynamically selects the scaling factor $\alpha$.

9. Use equation 3 , to embed the Watermark Image $\left(\mathrm{Y}_{\mathrm{i}}\right)$ into the cover image according to the predicted output of $\operatorname{OSELM}\left(\mathrm{Z}_{\mathrm{i}}\right)$ by using $\alpha$ obtained from step 8 .

$$
U i=Z i+\alpha \times Y i .
$$

10. Final Semi-Blind Watermarked image is generated.

\section{RESULTS ANALYSIS}

The proposed Algorithm is implemented in MATLAB by considering the authentic dataset. The performance of proposed algorithm is analyzed in terms of PSNR and MSE

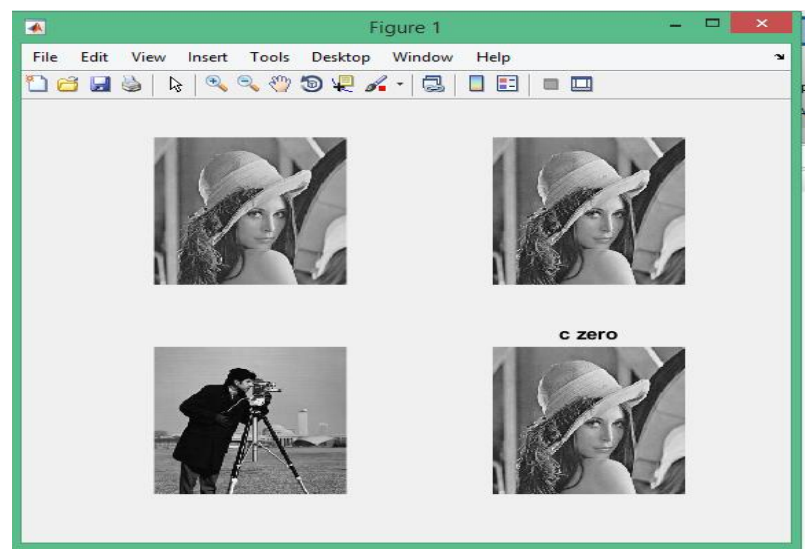

Fig 2: Watermark generation with proposed algorithm

As shown in fig 2, the GLCM and PCA algorithm is applied which select the scaling factor dynamically and according to that factor the watermarked image is generated.

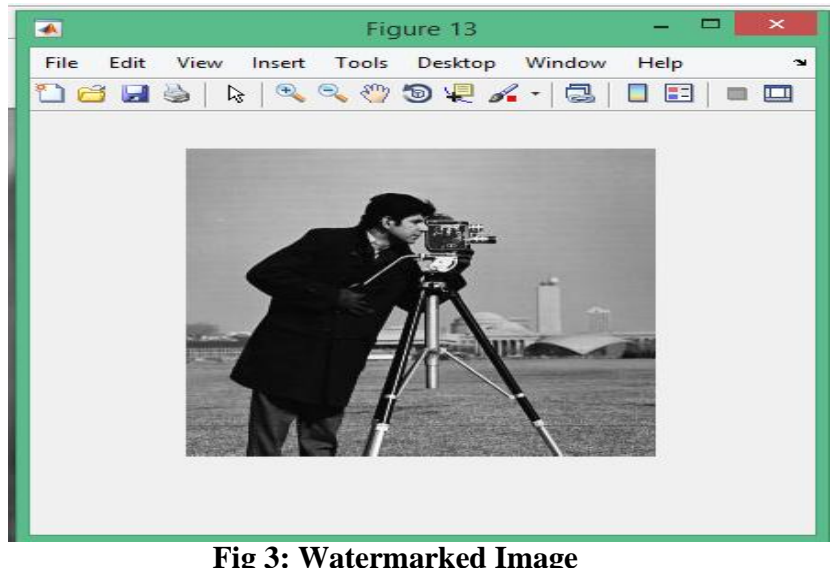

As shown in fig 3, the inverse operation is applied which will extract the watermark image and the original image.

In this paper we have shown the values of two image quality parameters which is defined as below:

\section{MSE \\ 2. PSNR}

In order to calculate PSNR, firstly we have to find out the value of MSE:-

\section{MSE}

MSE acronym for Mean Square Error which signifies the increasing squared inaccuracy between the original and compacted image. It is used for realistic purpose that permits to distinguish the actual pixel values of the non degraded image to corrupted image. The MSE basically gives an average of the squares of the faults between the original and degraded image. This fault is the quantity by which the pixels of the actual image fluctuate from the corrupted image.

Table 1: MSE comparison values
\begin{tabular}{|l|l|l|}
\hline Image & $\begin{array}{l}\text { DWT } \\
\text { Algorithm }\end{array}$ & $\begin{array}{l}\text { GLCM and } \\
\text { Algorithm }\end{array}$ \\
\hline Leena & 285.80 & 238.16 \\
\hline Taj & 275.93 & 229.94 \\
\hline Cat & 290.30 & 241.91 \\
\hline Bear & 292.45 & 243.70 \\
\hline $\begin{array}{l}\text { Mona } \\
\text { Lisa }\end{array}$ & 200.78 & 167.32 \\
\hline
\end{tabular}

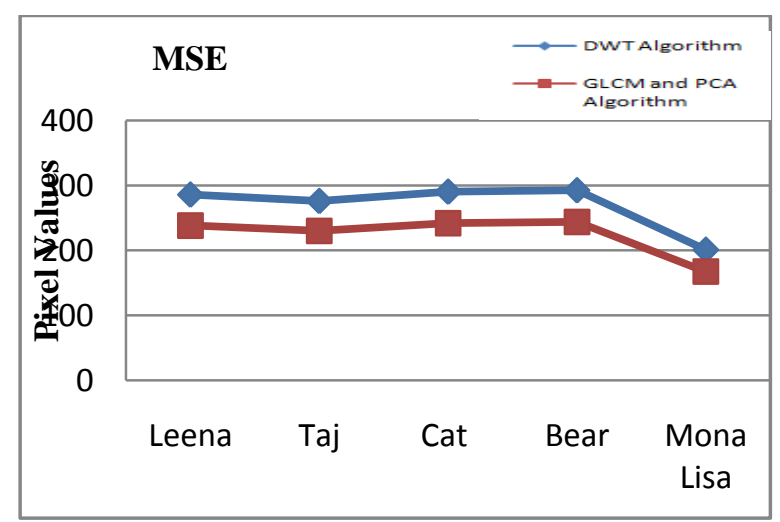

Fig 4: MSE comparisons 
As shown in the fig 4, MSE in the proposed and existing algorithm has been compared and it is been analyzed that MSE in the proposed technique lower value so there is less possibility of error.

\section{PSNR}

PSNR stands for peak signal-to-noise ratio that measures the proportion of highest or peak value of signal to the influenced value of distorted noise which has effect on the depiction quality. The PSNR metric calculates the peak signal-to-noise proportion within two images i.e. original and modified image. This fraction frequently measures the quality between the actual and a condensed image. If the value of PSNR is higher it means the reconstructed image had better quality.

Table 2: PSNR Comparison Values

\begin{tabular}{|l|l|l|}
\hline Image & $\begin{array}{l}\text { DWT } \\
\text { Algorithm }\end{array}$ & $\begin{array}{l}\text { GLCM and PCA } \\
\text { Algorithm }\end{array}$ \\
\hline Leena & 20.33 & 24.40 \\
\hline Taj & 20.46 & 24.55 \\
\hline Cat & 20.27 & 24.33 \\
\hline Bear & 20.25 & 24.30 \\
\hline $\begin{array}{l}\text { Mona } \\
\text { Lisa }\end{array}$ & 21.61 & 25.93 \\
\hline
\end{tabular}

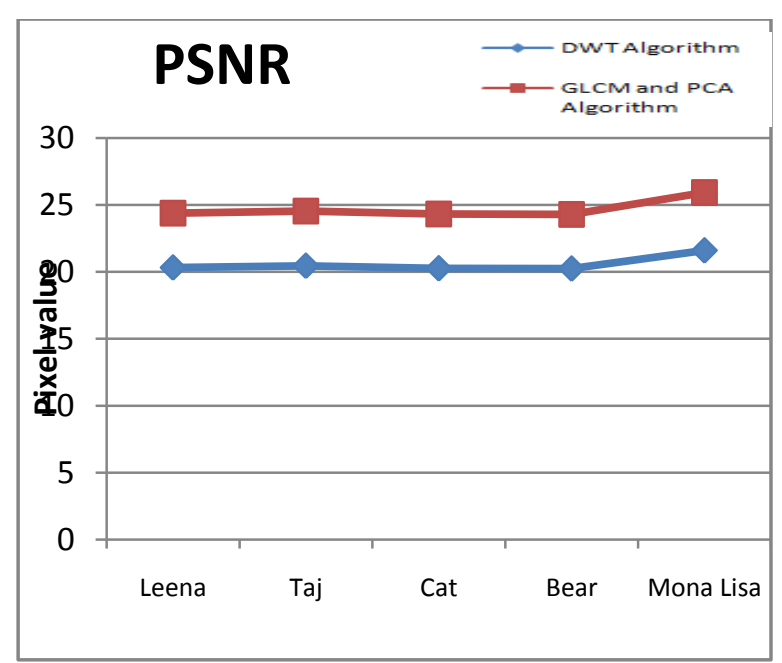

Fig 5: PSNR comparisons

As shown in the fig 5, PSNR in the proposed and existing algorithm has been compared and it is been analyzed that the value of PSNR in the proposed technique is higher than the previous work which show better results of image watermarking.

Table 3: Performance Comparison

\begin{tabular}{|c|c|c|c|c|c|c|c|c|c|c|c|}
\hline & \multirow{2}{*}{$\begin{array}{l}\text { Parameter } \\
\text { values }\end{array}$} & \multicolumn{5}{|c|}{ Existing Algorithm } & \multicolumn{5}{|c|}{ Proposed Algorithm } \\
\hline & & Leena & Taj & Cat & Bear & $\begin{array}{l}\text { Mona } \\
\text { Lisa }\end{array}$ & Leena & Taj & Cat & Bear & $\begin{array}{l}\text { Mona } \\
\text { Lisa }\end{array}$ \\
\hline \multirow{2}{*}{$\begin{array}{l}\text { Watermarked } \\
\text { image }\end{array}$} & PSNR & 20.33 & 20.46 & 20.27 & 20.25 & 21.61 & 24.40 & 24.55 & 24.33 & 24.30 & 25.93 \\
\hline & MSE & 285.80 & 275.93 & 290.30 & 292.45 & 200.78 & 238.16 & 229.94 & 241.91 & 243.70 & 167.32 \\
\hline \multirow{2}{*}{$\begin{array}{l}\text { Contrast } \\
\text { Attack }\end{array}$} & PSNR & 20.31 & 20.47 & 20.78 & 20.24 & 22.61 & 24.38 & 24.56 & 24.93 & 24.29 & 27.13 \\
\hline & MSE & 287.03 & 275.07 & 252.55 & 293.18 & 152.30 & 239.19 & 229.22 & 210.46 & 244.32 & 126.92 \\
\hline \multirow{2}{*}{$\begin{array}{l}\text { Sharpened } \\
\text { Attack }\end{array}$} & PSNR & 16.51 & 18.22 & 20.74 & 20.04 & 22.71 & 19.82 & 21.87 & 24.89 & 24.05 & 27.26 \\
\hline & MSE & 820.34 & 511.66 & 254.99 & 309.46 & 147.90 & 683.62 & 426.39 & 212.48 & 257.87 & 123.25 \\
\hline \multirow{2}{*}{$\begin{array}{l}\text { Salt \& pepper } \\
\text { Attack }\end{array}$} & PSNR & 25.04 & 25.16 & 25.12 & 25.07 & 25.66 & 30.04 & 30.21 & 30.17 & 30.07 & 30.80 \\
\hline & MSE & 77.86 & 75.29 & 76.02 & 77.16 & 65.49 & 64.95 & 62.48 & 63.07 & 64.48 & 54.46 \\
\hline
\end{tabular}

As illustrated in table 3, In terms of PSNR and MSE the performance of the proposed and existing algorithms are compared. It is demonstrated that better performance is provided by the proposed method as compared to other methods.

\section{CONCLUSION}

In this paper, efficiency of the watermarking approach is concluded as it hides all the sensitive information which is stored in the form of images. Here GLCM and PCA algorithm has been utilized in order to improve the working capability of the neural network based watermarking technique. The extracted features of an image are selected by the PCA algorithm and the features of the original image are extracted by the GLCM algorithm. The scaling factor defines the output of the PCA algorithm which is used for implementation. On the basis of simulation results it is concluded that proposed algorithm performs well in terms of PSNR and MSE. In future the watermarked image is generated by implementing the SVD technique.

\section{REFERENCES}

[1] Kashyap, Nikita, and G. R. Sinha. "Image watermarking using 3-level discrete wavelet transforms (DWT)." International Journal of Modern Education and Computer Science (IJMECS) 4.3 (2012): 50. 
[2] T. Serre, L. Wolf, S. Bileschi, M. Riesenhuber, and T. Poggio, "Object recognition with cortex-like mechanisms," IEEE Trans. on PAMI vol. 29, no. 3, 2007.

[3] J.-K. Kamarainen, V. Kyrki, and H. K " alvi“" ainen, "Invariance properties of Gabor filter based features overview and applications," IEEE Trans. on Image Processing, vol. 15, no. 5, pp. 1088-1099, 2006.

[4] D. Gabor, "Theory of communication," Journal of Institution of Electrical Engineers, vol. 93, pp. 429-457, 1946.

[5] J. Ilonen, J.-K. Kamarainen, P. Paalanen, M. Hamouz, J. Kittler, and H. K" alvi" ainen, "Image feature localization by multiple hypothesis testing of Gabor features," IEEE Trans. on Image Processing, vol. 17, no. 3, pp. 311-325, 2008

[6] Singh, Surya Pratap, Paresh Rawat, and Sudhir Agrawal, A robust watermarking approach using DCTDWT, International Journal of Emerging Technology and Advanced Engineering (ISSN 2250-2459, Volume 2, Issue 8 (2012).

[7] Al-Haj, Ali, Combined DWT-DCT digital image watermarking, Journal of computer science 3, no. 9 (2007): 740.

[8] Mansouri, A., A. Mahmoudi Aznaveh, and F. Torkamani Azar. "SVD-based digital image watermarking using complex wavelet transform." Sadhana 34, no. 3 (2009): 393-406.

[9] Lu, Chun-Shien, and H-YM Liao. Multipurpose watermarking for image authentication and protection, Image Processing, IEEE Transactions on 10, no. 10 (2001): 1579-1592.

[10] Martin, Alberto, and Sabri Tosunoglu, Image processing techniques for machine vision, Miami, Florida (2000).

[11] Chaturvedi, Navnidhi, and S. J. Basha. Comparison of Digital Image watermarking Methods DWT \& DWTDCT on the Basis of PSNR, image 2 (2012): 1.

[12] D. Gabor, "Theory of communication," Journal of Institution of Electrical Engineers, vol. 93, pp. 429-457, 1946.

[13] J. Ilonen, J.-K. Kamarainen, P. Paalanen, M. Hamouz, J. Kittler, and H. K" alvi" ainen, "Image feature localization by multiple hypothesis testing of Gabor features," IEEE Trans. on Image Processing, vol. 17, no. 3, pp. 311-325, 2008.

[14] J. Ilonen, J.-K. Kamarainen, and H. K " alvi" ainen, "Fast extraction of multi-resolution gabor features," in 14th Int Conf on Image Analysis and Processing (ICIAP), 2007, pp. 481-486.

[15] E. Simoncelli, W. Freeman, E. Adelson, and D. Heeger, "Shiftable multiscale transforms," IEEE Transactions on Information Theory, vol. 38,no. 2, pp. 587-607, 1992. 